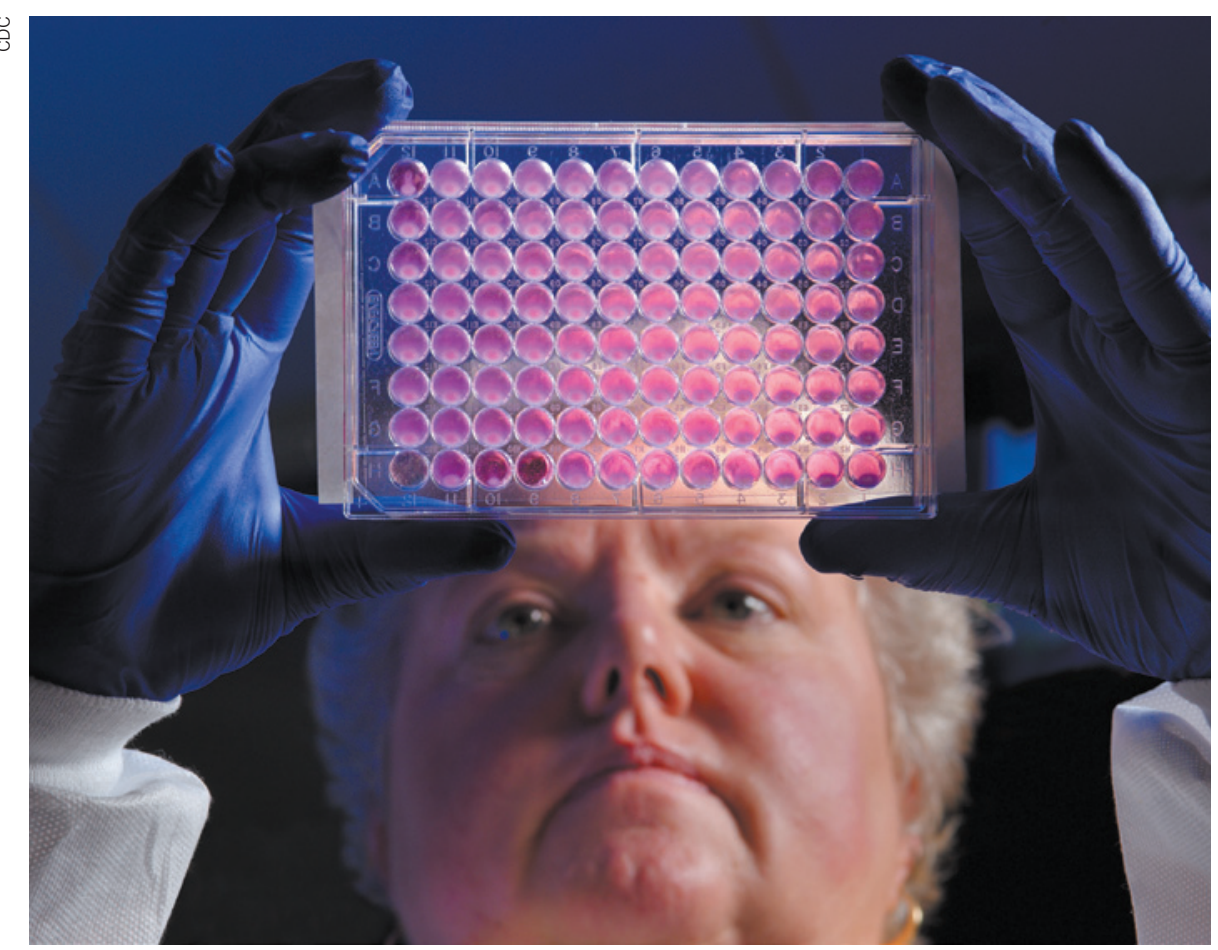

Tests for antibiotic resistance in bacteria require regular updates to remain effective as microbes evolve.

MICROBIOLOGY

\title{
Bacteria evade detection net
}

\section{Slothful response from regulators and manufacturers means antibiotic resistance is missed.}

\section{BY SARAH ZHANG}

$\mathrm{B}$ acteria that are resistant to almost all antibiotics are dreaded by physicians and patients alike. Finding such microbes in a hospital is bad enough, but failing to detect them can lead to something much worse: an outbreak.

That is why patients with bacterial infections are typically screened using automated lab tests that uncover the pathogen's identity and its susceptibility to various antibiotics. Because mechanisms of antibiotic resistance can pass rapidly between bacteria, especially in hospitals, the US Food and Drug Administration (FDA) periodically updates the rules for the machines used to perform these tests.

But there is a problem. The latest FDA rules have still not been fully adopted in tests for carbapenem-resistant Enterobacteriaceae, or CRE. This particularly worrisome group of pathogens can overcome carbapenem antibiotics - among the last lines of defence against resistant infections.

Each time the FDA updates its susceptibility criteria, manufacturers must upgrade the screening machines and resubmit them to the FDA for approval. Currently, none of the three manufacturers of such devices has incorporated the latest criteria for all three major types of carbapenem antibiotic.

Most hospitals continue to use machines with outdated software dozens of times

\section{"Making updates is a rather tortuous business."} a day. That means CRE cases are probably going undetected, says Janet Hindler, a clinical microbiologist at the University of California, Los Angeles. "It is sad this is a man-made problem," she says.

Identifying resistant bacteria matters both for surveillance and for individual patients, whose antibiotic treatments are tailored to the susceptibility of the infection, as determined by screening. The process uses a plastic plate with small wells containing up to a dozen antibiotics at different concentrations. The machine analyses bacterial growth in each well for 4-16 hours to determine the minimum amount of antibiotic needed to kill the pathogen. This antibiotic concentration, under the FDA rules, classifies the bacteria as susceptible, intermediate or resistant.

The resistant category keeps getting larger. One reason is that a better understanding of how drugs work in people - as opposed to in a Petri dish - shows that the threshold for antibiotic resistance in the body is lower than once thought. Another reason is that many types of resistant bacteria have emerged since various antibiotics were first approved. The FDA's drugevaluation section is currently updating the classifications for a backlog of 200 antibiotics.

The process of updating the US criteria for antibiotics starts with work by the Clinical and Laboratory Standards Institute (CLSI), a non-profit organization in Wayne, Pennsylvania, that collects drug data and helps to set the guidelines. After a decade of work on carbapenems, it eventually published revised classifications in 2010, including a larger resistant category for CREs. But the FDA did not start to implement its own categorization rules until 2012. The screening devices themselves are still out of date, so a bacterium considered resistant under the latest FDA criteria could still be reported as susceptible or intermediate.

Europe has removed at least one hurdle to getting its own up-to-date resistance categories. The expert body that recommends the classifications, the European Committee on Antimicrobial Susceptibility Testing, also sets the criteria for the European Medicines Authority. And companies can make small updates to their devices without having to reapply to regulatory bodies for approval, says David Livermore, a microbiologist at the University of East Anglia in Norwich, UK. In the United States, he says, "making updates is a rather tortuous business, particularly if the CLSI has changed its breakpoints and the FDA hasn't".

Manufacturers concur with Livermore. Before it will clear an updated device, the FDA sometimes requires further studies that can take two to three years to conduct and cost tens of thousands of dollars, says Bill Brasso, president of the Susceptibility Testing Manufacturer's Association and a scientist at one of the manufacturers, BD Diagnostics Systems in Sparks, Maryland. It can then take up to a year to get FDA clearance, he adds.

But Sally Hojvat, director for the division of microbiology devices at the FDA, says that data from earlier studies will often suffice. Even when new studies are required, she says, they involve only the analysis of bacteria, not large clinical trials. Sometimes the process drags on because manufacturers do not submit all the information the FDA needs, she adds.

Neither manufacturers nor the FDA has pushed for timely updates, says Amy Leber, director of the microbiology lab 
- at the Nationwide Children's Hospital in Columbus, Ohio. "The manufacturers point their fingers at the FDA, and the FDA points its finger at the manufacturers," she says.

Leber found her own way around the problem. Last year, her lab colleagues hacked into her hospital's machine to upgrade the software that governs resistance categories for carbapenems. Earlier this year the machine identified a case of CRE. The patient was swiftly isolated and an outbreak averted.

Leber worries about smaller clinics that do not have advanced screening equipment, let alone the time and money to update their devices. Meanwhile, CRE cases are spreading out of urban centres. Since the microbes were first detected in North Carolina in 2001, they can now be found in nearly every US state.
Jean Patel, deputy director of the office of antimicrobial resistance at the Centers for Disease Control and Prevention in Atlanta, Georgia, wants screening devices updated faster so that her agency can conduct better surveillance. "It has been a little frustrating to watch how long this has taken." -

Additional reporting by Elizabeth Gibney.

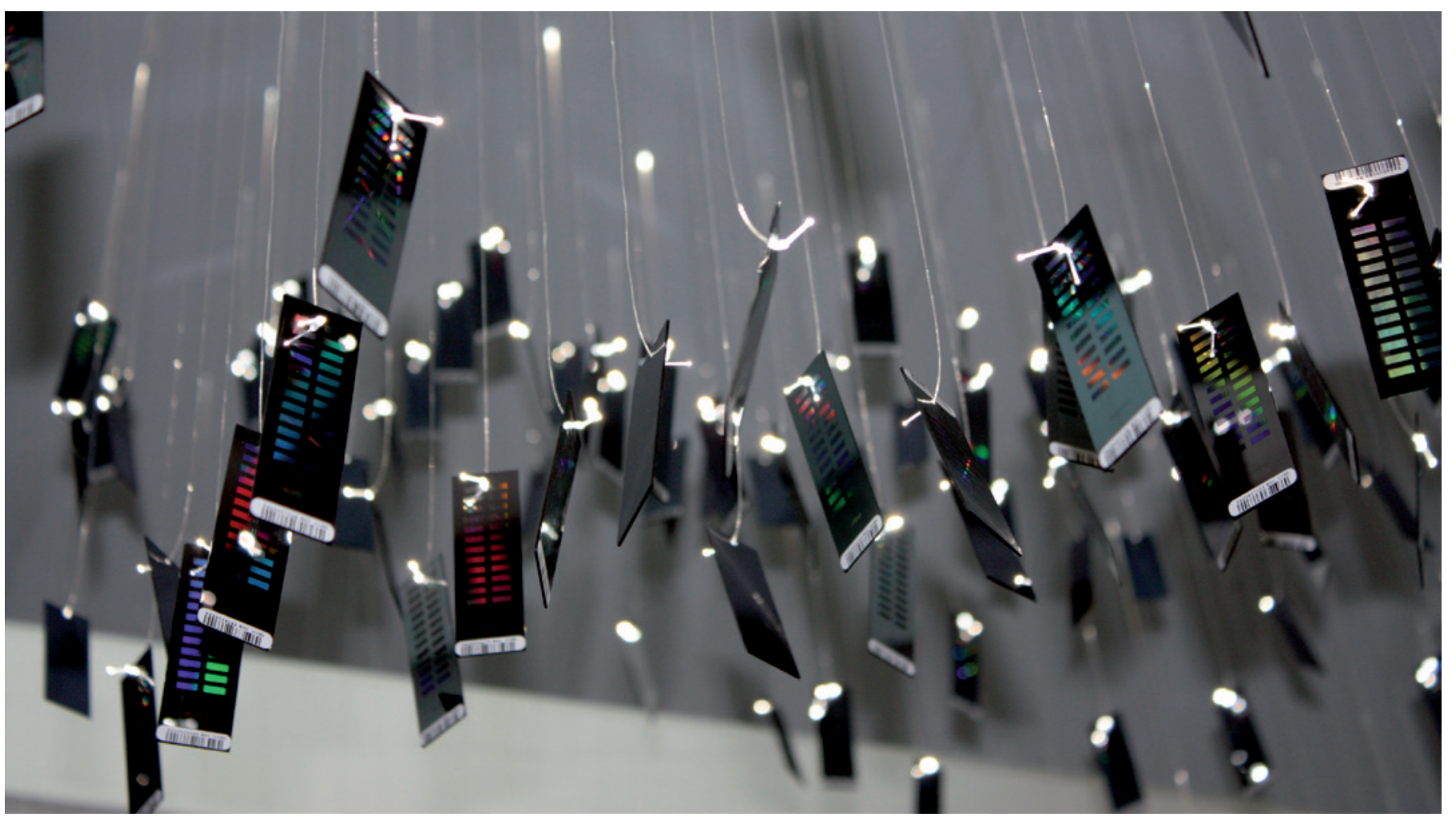

Illuminated microarrays formed part of a display at a medical museum in Copenhagen in 2011.

\section{Museums hunt for relics} from genomics' early days

\section{Collectors band together to salvage cast-off equipment.}

\section{BY HEIDI LEDFORD}

$\mathrm{I}$ $\mathrm{n}$ a former envelope factory sits a boxy grey and blue machine the size of an oven - the tenth acquisition this year for a Massachusetts science museum. It is a colony picker, a robotic arm that plucks bacteria from Petri dishes and drops them into a tray with 96 wells, from which DNA is extracted, amplified and sequenced.

At the start of this century, the device powered genomics research at the Whitehead Institute for Biomedical Research in Cambridge, Massachusetts. Now retired, it rests in the warehouse of the Massachusetts Institute of Technology (MIT) Museum, sandwiched between a sewing machine from around the 1920s and an analog computer from the 1950s.

Genomics researchers

\section{$\rightarrow$ NATURE.COM}

For a slideshow of

equipment sought by curators, see:

go.nature.com/05ruqh might find it hard to believe that museum patrons would be interested in paying homage to a piece of lab equipment as pedestrian as a colony picker. Curators of science and technology museums say that is exactly the problem.

"Very few scientists have any idea that they should preserve these things," says Thomas Söderqvist, director of the Medical Museion in Copenhagen. "They just throw them out."

But in the past two years, a confederation of about a dozen science museums has 